\title{
8 Reach, Bracket, and the Limits of Rationalized Coordination: Some Challenges for CSCW
}

\author{
Elihu M. Gerson
}

\subsection{Introduction}

There has been a long-term shift in the way computing arrangements support other kinds of work. The first generation of computing technology speeded up and automated the performance of standardized repetitive tasks, such as payroll preparation. Later generations concentrated on supporting and augmenting varying but routine tasks: Desktop systems running "productivity" applications such as word processors and spreadsheets were typical applications. ${ }^{1}$ More recently, the development of the World Wide Web, broadband communications, wireless technology, standardized markup languages, and other innovations have created many new contingencies and possibilities. Computing has become part of the day-to-day and even minute-to-minute flow of activities, and significant computational capacity is becoming ubiquitous. As a result, attention has increasingly shifted to understanding and supporting communication and cooperation among people, rather than automating specific tasks. The focus is thus now on helping people deal with one another conveniently, and performing loosely defined tasks well enough. This requires allowing for unpredictable contexts and changing needs.

These trends have two major implications for the relationship of computing and work. The first implication is hyper-distribution. For many years, the movement of computing capacity from centralized installations in large organizations to smaller installations in smaller organizations

\footnotetext{
${ }^{1}$ The history of computing is a substantial area of scholarship in its own right. Two useful overviews are Campbell-Kelly (2003) and Ceruzzi (2003). The development of information systems in the years before the emergence of computers is covered in Yates (1989).
} 
changed the basic relationships between computing and work incrementally, and in predictable ways. Recently, however, we have crossed a threshold in the ways information systems support work. Joint activities are now easily conducted across organizational boundary lines. Distancerelated barriers to coordination and cooperation are also decreasing quickly. While face-to-face interaction is still needed for many purposes, steady progress in the quality and cost of telecommunications is steadily chipping away at the need for meetings in which people are physically present together.

The second implication of these trends in computing is hyperaccessibility. An increasing number of objects and tasks are becoming capable (at least in principle) of finding and communicating with one another across distances, jurisdictions, and organizational boundaries. For example, it has already become routine for automobiles to report their locations, a capacity that gladdens the hearts of car rental firms, law enforcement agencies, and perhaps the parents of teenagers. The emergence of Bluetooth and RFID technology raises the possibility that we will shortly see extensions of this trend, as individual objects begin to interact with the places and organizations that contain them. While this trend certainly provides opportunities for improved coordination, new services, and many other benefits, it also entails many new or increased difficulties in managing privacy, security, accountability, and reliability.

"Hyper-distribution and hyper-accessibility" is an awkward phrase. We need a convenient term to refer to the distribution of tasks across organizational, spatial, and temporal boundaries. I'll call this the "reach" of a task. Increased reach is having many important consequences for the organization of work. It is removing or reducing many kinds of constraint and thus makes new arrangements feasible. At the same time, it is creating new problems that require new kinds of constraint. For example, a mobile telephone with a built-in camera and Internet access is a wonderful thing when I'm in the store and I want to ask my wife which model widget we should buy, but I don't want someone following me into the public restroom with one.

As a result of these trends, many activities that were conducted on a person-to-person basis no longer have the immediacy that lets people see and hear what other participants are doing. One example is the loss of emotional context when using e-mail and instant messaging rather than face-toface or even telephone communication. Much important information about mood and temper is lost. More generally, the trend implies a loss of context for many kinds of interaction. Increasingly, interacting parties don't have a good knowledge of one another's history and circumstances. This limits the trust which parties can place in one another. Coordinating tasks 
in these circumstances means that formal mechanisms such as passwords, ID cards, and credit checks are needed to assure participants of reliability and safety. Interaction is thus becoming explicitly controlled, formal, and mediated by a variety of devices and procedures. Increasing reach also means that work is becoming more intensively computer-supported. $\mathrm{CSCW}$ research is thus becoming relevant, in principle, to almost every kind of work. This increased relevance requires careful thinking about the new ways in which work is performed, how tasks interact, and how work interacts with information systems.

\subsection{Coordination Mechanisms}

Increasing reach implies that the problem of coordinating tasks and people (never easy) becomes markedly more complex. Resources and results must be brought together at appropriate times and places if the work is to succeed. Of course, there are many ways of addressing this question; it is the defining problem for research in management science. Two classical treatments are Lawrence and Lorsch (1967) and Thompson (1967). Recent research in several disciplines emphasizes relations among organizations as well as within them. ${ }^{2}$ Malone and Crowston (1994) argue for an emerging research area focused explicitly on coordination, which they define as the management of dependencies among activities. Here, I want to use a different definition of coordination, one focused on the ways in which the parts of an activity function (or fail to function) together. In particular, I am concerned with the implications of increasing reach for the cofunctioning of tasks. The question for $\mathrm{CSCW}$ is: What role does computing technology play in this process? The very general and powerful notion of "coordination mechanism" proposed by Schmidt and Simone in 1996 helps us specify the issues involved in a useful way:

A coordination mechanism is a specific organizational construct, consisting of a coordinative protocol imprinted upon a distinct artifact, which, in the context of a certain cooperative work arrangement stipulates and mediates the articulation of cooperative work so as to reduce the complexity of articulation work of that arrangement. (Schmidt and Simone 1996, p. 180; emphasis in original)

Schmidt and Simone define coordination mechanisms in term of distinct artifacts. The prototypical artifacts of this kind are business forms such as bills of lading or restaurant checks. An alternative approach, adopted here,

\footnotetext{
${ }^{2}$ See, for example, Best (1990), Dimaggio (2001), and Garud et al. (2003).
} 
is to think of coordinating mechanisms as tasks dedicated to orchestrating the work of other tasks. Often, this work makes use of objects that serve as a focus of the interaction-typically, business forms that carry information from one task to another and back again. Our primary concern, however, is with the work that meshes two or more activities. Hence, it's useful to think of a coordinating mechanism as consisting of both an artifact and the work of using it-e.g., filling out and interpreting forms "correctly." For example, a restaurant check serves to coordinate the interaction of server with patron, server with kitchen staff, and patron with cashier. This works, of course, only if everyone uses the check in the way it was intended. So the work of preparing and using the device correctly is a part of the mechanism, which is best considered as a specialized kind of task.

The idea of coordinating mechanism is motivated by the idea of articulation work developed by Anselm Strauss. Strauss used the notion of articulation work in two different senses (e.g., Strauss 1987). On the one hand, articulation work is about making sure all the various resources needed to accomplish something are in place and functioning where and when they're needed in the local situation. This means bringing together everything needed to accomplish a task at a particular time and place, including all the administrative and support functions such as janitorial services, food service, equipment maintenance, and covering for staff out sick or on vacation. The concern and emphasis in this sense are on particular situations rather than classes of activity.

In its second sense, articulation work means "putting together tasks, task sequences, task clusters-even aligning larger units such as lines of work and subprojects-in the service of work flow" (Strauss 1991, p. 100). In this second sense, the focus is not so much on the specifics of work in a particular local situation as it is on making sure that different kinds of activity function together well. The two senses, of course, overlap heavily-especially when all the tasks are part of the same organization and are carried out in the same place.

The notion of articulation has been used in the CSCW literature most often in the second sense, which I'll call metawork-the work of making work go well. I'll keep the term "articulation" (or "local articulation") to refer to the first of Strauss' senses. We can always distinguish articulation work from metawork because articulation work is about a particular situation. So, for example, the supplies cabinet in every office has to be restocked from time to time. Restocking as articulation work consists of putting needed supplies in a particular cabinet on a particular date. Metawork, by contrast, consists of specifying what restocking consists of. It's the distinction between specifying what goes on a preprinted shopping list and checking off the items on a copy of the list for today's shopping trip. 
The distinction between the two senses isn't very important when we're focused on single organizations, as Strauss did in his 1987 article. There, he explicitly set aside the problems associated with analyzing articulation of single projects across multiple organizations, multiple worlds, and multiple sites. The importance of increasing reach, however, means that we must make the distinction clear and understand its implications. The work of specifying the work to be done is one thing; the work of ensuring performance in specific circumstances is another.

The analysis of coordination mechanisms then, is primarily about metawork-although, as we shall see, articulation has an important part to play as well. A coordination mechanism is something that activities use to orient their courses vis-à-vis one another. It is thus part of the workflow in two different activities simultaneously and serves to make them contingent upon one another. A coordinating mechanism may be as simple as a job ticket, on which the progress of a joint task is marked for all participants, or it may be a complex activity in its own right.

"Coordination mechanism" is another awkward term, especially since I am going to be discussing coordination in other senses below. Hence, I will adopt the term "bracket" as a synonym for "coordination mechanism." The significant things about a bracket (or bracketing as an activity) are (1) it connects two things together and makes them part of a larger system of dependencies. (2) it does so in specific ways. and (3) it also holds them apart and keeps them distinct. I mean all of these senses to apply when I write here of brackets. I am trying to call to mind not only the specialized punctuation marks that often signal the presence of metawork [thus], but the pieces of metal that fasten and orient objects to one another.

\subsection{Reducing the Complexity of Metawork and Articulation: Varieties of Rationalization}

In the view of Schmidt and Simone, the function of a coordinating mechanism is to reduce the complexity of metawork and articulation work-to simplify the ways in which the parts of a system interact. An effective mechanism thus helps to rationalize the interaction of the coordinated tasks. I use the term "rationalization" here in the economist's sense of doing more with the same resources, or the same work with fewer resources. This seemingly simple idea turns out to be fairly complex, because there are three kinds of rationalization, and sometimes they trade off against one another. 
One kind of rationalization is segregating rationalization. This makes things independent of one another, removing the connections or contingencies among them wherever possible. Complex tasks are broken into multiple independent tasks. Similar things are grouped, and dissimilar things are segregated. Things are treated as unrelated members of a set, rather than as parts of a whole. Finally, relationships among things are treated as unary properties of their constituent units.

The second kind of rationalization is standardizing rationalization. This makes the connections and relationships among things uniform. Standardization substitutes repetitive similarities for unique or diverse activities, materials, tools, and situations. In doing so, it makes dealing with them cheaper, more convenient, and more reliable.

The third kind of rationalization is coordinating rationalization. This works by fine-tuning and refining relationships so that they are particularly well-suited to their situation. Within a given activity, tasks are made more responsive to one another by removing everything that does not contribute directly to smooth functioning and by strengthening everything that does. Frequently (but not necessarily) it means taking advantage of specialized local circumstances or knowledge in order to create specialized local short-cuts.

All three ways of rationalizing simplify relationships: by removing them, homogenizing them, or refining and specializing them. All three ways of rationalizing make activities more efficient; more gets done with the same resources, or it takes fewer resources to accomplish a given task.

Sometimes, the different rationalization processes work together or reinforce one another. For example, increasing standardization of raw materials and parts makes it far more practical to segregate manufacturing steps that assemble the parts into finished products. This was precisely the advantage of the American System of manufactures, introduced in the 19th century (Hounshell 1984). In the older system, parts were hand-fitted together by skilled craftsmen. In the American System, parts were manufactured in high volume to close tolerances, so that they were interchangeable. As a result, assembly of the finished product required much less skill.

Standardization of materials and parts means that different manufacturing steps can be segregated temporally, spatially, and organizationally. The performance of each task can in turn be standardized, e.g., via time and motion engineering (cf., e.g., Kanigel 1997). Henry Ford's mass production technology was built on the use of such standardized tasks performed on standardized parts and materials (Hounshell 1984).

Rationalized work processes produce standardized products as well. Sometimes this is of value to end users. When you're in a city far from home, for example, the presence of familiar franchised stores tells you a 
great deal about the quality of goods available. Sometimes, of course, standardization is a drawback because it reduces choice, but for intermediate or producers' goods standardized inputs means simpler production processes and lower costs in the next stage of production. Segregating and standardizing rationalization thus tend to reinforce one another. Segregation makes it easier to specify and enforce standards, while standardization makes it easier to segregate functions in different organizational units. Standardizing and segregating rationalization are often discussed together under the rubric of "modularity" (e.g., Garud et al. 2003; Sturgeon 2002).

Segregating and standardizing rationalization together often trade off against coordinating rationalization. The latter is aimed at making parts work together as efficiently as possible, minimizing waste and friction in the interests of a well-adapted system. This means fine-tuning interacting parts so as to make them accommodate one another's peculiarities. In doing so, coordinative tasks typically take advantage of local circumstances that offer opportunities for lower costs. Such advantages, which economists call "positive externalities" (e.g., Cornes and Sandler 1996), are often incorporated into the production process. Warehouses, for example, tend to be located where transportation facilities come together.

The specializing local character of coordinative rationalization is often in direct conflict with standardizing and segregating processes. For example, in writing computer programs, it's often possible to improve the performance of a program by taking advantage of idiosyncrasies in the design of the hardware or operating system on which the program is running. This form of coordinative rationalization is typically deprecated, however, because it means that the program will run less well (or not at all) on other hardware or operating systems. Segregating and standardizing rationalization, by contrast, seek to improve overall efficiency by removing dependencies or interactions among parts, and grouping each separate kind so that they can be processed uniformly at relatively low unit cost. They try to ignore or overcome local circumstances and make work processes less dependent on externalities. In short, segregating and standardizing rationalization tend to reduce or eliminate the costs of coordination.

\subsection{The Role of Protocols in Rationalization}

Segregation and standardization eliminate many kinds of local coordinative arrangement, but they do not eliminate the need to coordinate. Rather, they put it on a different basis. For example, if we employ a system of interchangeable parts to replace an artisan who fits variable parts together by 
trimming them, we need a system to ensure that the parts used are similar enough to make interchangeability practical. This typically means that new ways of coordinating work elsewhere in the production process-new kinds of metawork-must be implemented to compensate for the eliminated artisan. As I noted above, these new procedures tend to be formal, explicit, and standardized. They are, in fact, brackets aimed at replacing one way of coordinating work with another. More precisely, casual articulation work rooted in local and personal arrangements ("Honey, can you pick up some milk on the way home tonight?") is replaced with explicit coordination via formal mechanisms (the refrigerator adds milk to the shopping list, which is automatically transmitted to the supermarket via the Internet).

That is, increasing segregation and standardization eliminate some kinds of coordination work, but they also provide the basis for new kinds of coordinative rationalization. Segregation and standardization may work against coordination within the scope of a particular activity, but they also enable new forms of coordination among activities as well. It is important to note two things about this process. First, local articulation is typically replaced by metawork. One example, becoming common now, is automatic updating of software on personal computer systems. Rather than ask users to keep track of which updates are needed, and when, and (perhaps) doing it themselves, perhaps correctly, an automatic process carries out this work. Second, the new kinds of coordinative rationalization are typically associated with increased reach over larger organizational, geographic, and temporal scales than was the case previously. In other words, if people can be convinced to do things the same way, then knowledge of this similarity enables more effective (and/or simpler) coordination mechanisms. The widespread adoption of cheap, reliable clocks and watches, for example, made it possible to schedule work and other events with great accuracy (Landes 1983). This, in turn, facilitated the emergence of many new forms of social organization.

The new kinds of coordinative rationalization are created through the development of standardized arrangements-protocols-that support metawork across many situations. Protocols abstract from many similar situations and specify a class of brackets or the mechanism for generating a bracket. The abstract character of protocols is critical, because abstraction means brackets can be represented, compared, combined, and manipulated as programs and data. This characteristic, in turn, enables coordinating many different sorts of tasks by similar means. Nothing illustrates this principle better than the Web itself, which answers almost any question by using standardized search services to supply appropriate URLs. 
Protocols have become increasingly common and important as the Internet has developed; in a real sense, the success of the Internet is based on protocols such as TCP/IP, XML, SOAP, and RSS. In fact, in the last 10 years, specifying and exploiting new protocols have probably become the most important ways of creating large-scale innovation in the industries concerned with information systems.

Protocols, with their standardized way of representing and interpreting information, provide a basis for replacing specialized local knowledge with general procedures. Using protocols means making things work in comparable ways in many situations. Tasks conducted in accordance with a protocol function adequately with a variety of other such tasks. They do this without imposing the need to worry about how others have their work arranged, and without respect to organizational boundaries.

Protocols thus enact a sharp distinction between form and content. Much of the metawork of intertask coordination can thus be specified and organized without considering much of the detail of specific local circumstance. Typically, this is accomplished with the use of markup languages, application programming interfaces, and similar arrangements. Abstract protocols, with their focus on form rather than content, provide the basis for new kinds of bracket to organize the metawork associated with increased reach. Such protocols are particularly valuable because increased reach means that there more situations to be considered, and hence more variety and uncertainty among local circumstances. The use of abstract protocols is not just a matter of inventing new standardized parts, like adding a new structural element to an erector set. Rather, it is like inventing a new class of objects that connect things that could not be connected before, such as a new device that allows reliable connections between erector set parts and Lego blocks.

Consider, for example, some uses of airline flight information made practical by the growth of the Internet. Airlines and traffic controllers have always tracked flights in the air, and estimated arrival times have been available to the public via telephone for many years. Access to flight information via the Internet means that it's now possible for anyone to follow the pattern of arrivals and departures at local airports automatically. With this information, firms in the travel industry can coordinate their own work with airline flight times. Hotels can be aware that expected guests are arriving late. Limousine, shuttle, and taxi firms can get similar information for particular arrivals. They can also use local ground traffic monitoring systems to estimate changes in the flow of traffic to and from the airport, and thus improve their dispatching. This capacity is further improved if they can make use of location information (via GPS) from their own vehicles as well. 
Abstract protocols thus permit many different sorts of systems to coordinate in ways that were previously impractical. For example, nobody ever actually wrote software to tie information about any hotel's room inventory to every airline's flight schedules. Yet every hotel now can do this. This same abstraction and generality also mean that many previously difficult local problems can now be solved easily. At the airport, for example, it is now much easier (in principle) for drivers and passengers to meet reliably despite bad weather, flight delays, traffic jams, and not knowing one another. Drivers and arriving passengers can now exchange photos and detailed directions for meeting via cell phone as passengers disembark.

These abstract protocols can be combined fairly easily to create applications (i.e., brackets) that did not exist before. This increase in standardized mix-and-match capacity means that people can come up with new arrangements that work across organizational boundaries, without the prior approval or even knowledge of their managements. Moreover, their innovations aren't confined to a single organization; often, new arrangements readily become available to everyone. This can have unexpected consequences on a large scale, as when the increased reach created this way forces changes on an entire industry. The clearest example of this to date is the emergence of peer-to-peer ("P2P") file sharing and its effect on the music recording industry.

For the most part, the new brackets enabled by protocols depend on the same computing innovations that are giving rise to increasing reach. Instead of relying on the close integration of local circumstances, as articulation arrangements have traditionally done, they tend to be abstract and standardized. They also tend to rely on metawork rather than local articulation work. But the local articulation work must still be done.

\subsection{Local Articulation and the Limits of Rationalization}

It's often difficult to rationalize (or even change) some local arrangements. Local problems are typically solved opportunistically. Casual improvisation over time becomes entrenched as local conventions, which are typically organized around the skills and tastes of particular people. As a result, local articulation arrangements are typically fine-tuned to particular situations and are dependent upon particular individuals and circumstances. Over time, new arrangements are added, each making use of idiosyncratic local conventions and further entrenching them. For this 
reason, local arrangements can be extremely effective, but it can be very difficult to understand or modify them. ${ }^{3}$

Local circumstances always have complexities that cannot be captured in a formal system, no matter how elaborate or forgiving it is. Hence, even as brackets are built to support local articulation work, there is always some articulation work beyond their reach. Well-designed information systems can help with these problems, but they can't eliminate them. That is: You can't build a repair kit that can anticipate all possible failures, and even if you could, you can't guarantee that it won't break just when it's needed. An important challenge for $\mathrm{CSCW}$ then is understanding the nature of these limits.

The difficulties of doing so are greatly exacerbated by increasing reach, which forces a significant change in the notion of "local." Traditionally, "local" referred to a particular organizational and/or geographic setting. With increasing reach, however, it is now necessary to consider the joint performance of single activities or workflows as "local" even though cooperating participants might be geographically and/or organizationally distributed. That is, "local articulation" (including "task-local" as well as "place-local" and "office-local") should refer to all the activities that require attention if a given task is to be carried out properly. Increasing reach means that colleagues may be interacting in different times of their daily cycles, or in different climates, or with different cultures, or under different organizational policies or even legal systems. Moreover, they will be supported by different administrative services: computing, food, security, medical, janitorial, etc. Indeed, these local contexts might be changing as participants move between office and home, or from office to limousine to airport to plane and back.

With "local" more complicated than it used to be, analyzing and supporting articulation work has acquired new contingencies. Can brackets to support local articulation be built? What limits are there on doing so? In order to deal with these issues, it's necessary to refine the idea of local articulation work. Analytically, local articulation is really several different kinds of problem. I'll discuss two of them here: customization, which accommodates the particular and local to the general and global; and reconciliation, which accommodates the demands of different local stakeholders.

\footnotetext{
${ }^{3}$ Becker (1982) provides a sociological analysis of entrenched practices. The philosophical foundations are discussed by Wimsatt $(1986,2001)$.
} 


\title{
8.6 Customization: Accommodating the Particular and Local to the General and Global
}

\begin{abstract}
protocols present slots that must be filled in with appropriate local information. Providing this information is not necessarily a simple task; it requires interpretation on both sides in order to ensure suitable entries. For example, designers of data entry modules have long since learned to provide explicit alternatives wherever possible [e.g., "Sex: $(\mathrm{M} / \mathrm{F}) "$ rather than "Sex: ___, templates to guide entry (e.g., "mm/dd/yyyy" for date fields)] and help in order to ensure that data are entered in appropriate forms. Of course, the guidance provided must itself be reasonable from the viewpoint of those providing the information. One kind of local articulation work thus consists of figuring out what the appropriate information is, gathering it, formatting it, and providing it. These tasks can prove to be formidable challenges in their own right. The difficulty people often face in filling out even relatively simple tax returns is a well-known example.
\end{abstract}

Of course, successful articulation consists of more than figuring out how to fill out forms effectively. This is important, but it is dependent on the coherence of the conceptual schemes used on both sides of the bracket. Where such schemes are known to all concerned, conventional, and commensurate with everyone's views and purposes, the problems of interpretation will be relatively minor. This does not mean that the consequences of a failure will be small. For example, accidentally interchanging metric and English units of measure is a small problem, in that it can be fixed quickly and cheaply if discovered in time. But the consequences of not noticing and fixing such an error can be very large. In 1999, for example, the Mars Climate Orbiter satellite was lost as a result of just such an error (NASA 1999). Where schemas are not understood, or not yet conventional, or incommensurate, the potential for some sort of failure increases. The chances of this happening increase with reach across task, organizational, cultural, and geographic boundaries. So one major challenge to CSCW research is finding means of recognizing and describing when conceptual schemes are out of line with one another.

Customization also includes the work of making equipment and materials fit with local procedures. Strauss' original thinking about articulation work was motivated in part by his observation of health professionals working with specialized equipment in hospitals to make it fit the needs of particular cases (Strauss et al 1985). Similar kinds of problem have been addressed by $\mathrm{HCI}$ and CSCW researchers studying how computer users "tailor" their software to fit well with local needs (e.g., Trigg and Bødker 1994). 
Customization problems have concerned systems developers for many years. Indeed, such problems are at the heart of traditional research on computer-human interaction. The work of analyzing customization problems is worsened and sometimes transformed by increasing reach because traditional systems development has been done either within (and for) single organizations or in ways that ignored local circumstance. Increasing reach means that systems interact with organizations and people in more intimate ways, at the same time that there are more opportunities for more things to go wrong in a greater number of ways. Such failures can easily lead to disaffection and conflict, thus turning problems of customization into problems of reconciliation.

\subsection{Reconciling Local Participants}

Most local articulation work consists of dealing with normal, expectable contingencies of coordination: The copier runs out of toner, someone calls in sick, an instrument needs calibrating, a customer is complaining in a routine way about an ordinary problem, somebody has left the dirty coffee pot on the burner again - the list is endless. Typically, there are established routines-brackets-for handling these contingencies. These may be as simple as "while you were out" message slips or fax cover sheets, or they may be elaborate procedures involving many people. Sometimes, these routines fail because the requirements imposed by one procedure come into conflict with those expected by another. When this happens, a bracket can reveal or become enmeshed in conflicts that were tacit or latent before it was installed. Indeed, the major point of one study of articulation work was that arrangements for coordinating are themselves often the battlefields upon which contending participants work out their claims (Gerson and Star 1986).

This is the general reconciliation problem: Where there are multiple contending parties with differing viewpoints, interests, and concerns, some means must be found to reconcile their differences if their interaction is to continue. Sometimes, there is a mechanism in place-an authority-that can adjudicate differences. In high-reach situations, this is unlikely. Differences must be resolved by means that do not rely on appeal to authority. This is accomplished by aggregating the preferences of participants to form some kind of collective decision or policy that is reasonably efficient, effective, and equitable. Of course, choosing the governing criteria of efficiency, efficacy and equity is itself a reconciliation problem. 
Consider the hypothetical example of a session at a scholarly meeting held in mid-winter at a hotel. As people come into the room, the room queries their personal computers or PDAs for their temperature preferences. The room then sets the room temperature to reflect the joint preferences of the attendees. From a technical point of view, this is a trivial problem: One chooses an algorithm to aggregate the individual preferences, expresses it in a convenient programming language, and passes the result to the building's climate control system. However, any algorithm for aggregating preferences inevitably favors some preferences (or rather, preference holders) rather than others, so the issue of fairness necessarily arises. More generally, local conflicts are often reflected in decisions about bracket design and use. This is more likely to occur with increasing reach, if only because there are more kinds of local interest to reconcile.

Increasing reach means that the flow of everyday work thus comes to look like work in occupations (for example, medicine, sales, law, research, organized crime, and prostitution) where reconciliation problems are not always anticipatable. This is especially true where the client can't be commanded, but must be persuaded to cooperate. This is a very important point, because it makes for a qualitative difference in the kinds of problemsolving approaches that might work well. Tasks whose conduct crosses jurisdictional boundaries (whether organizational or territorial) don't have a ready formal or official means of reconciling differences. A disagreement among people in the same organization can be resolved by their common management, but a comparable disagreement across organizational boundaries only becomes more complex, more expensive, and no more certain of resolution, as managements become involved. This is a critical difference between systems oriented toward single participants and those oriented toward multiple independent participants. The means for resolving conflicts safely and reliably are very different. In these circumstances, support for local articulation means providing tools and procedures for working with complex negotiations. There can be no general guarantee that these must be successful, or that agreement must be reached. Of course, the need to reconcile contending concerns is a consideration in every circumstance. But increasing reach intensifies and extends the problem in important ways.

Understanding this class of problems is a matter for basic research in all of the social sciences. One important and provocative approach to these problems comes from institutional economics, which has a significant line of work focused on these concerns. This has grown from the realization that the cost of shopping is a significant part of the costs of acquiring needed resources (Coase 1937; Williamson 1975, 1996). From this insight has grown an approach that examines the tradeoffs between producing 
something "in house" on the one hand, and purchasing it on the other. Where there are few qualified suppliers (e.g., as when local knowledge is especially important), for example, producing a needed resource in-house becomes more attractive.

Most of the conditions favoring use of the market, rather than in-house production, can be expressed in terms of the required metawork and articulation work. When needed metawork is standardized and readily available for purchase, and when local articulation work is easily and reliably done, then the appropriate coordination mechanisms are ordinary arrangements of buying and selling: proposals and quotes for fixed-priced contracts, delivery schedules, and so on. When articulation work becomes complex and unreliable, and/or when metawork is not standardized, then the necessary negotiations are much more complex, and are more reliably handled in the context of a common administrative regime.

Models of each of the two major organizational forms, markets and hierarchies, have their strengths and weaknesses. Neither group of models can deal very effectively with the problems raised by increasing reach. This difficulty has spurred a search for other, intermediate, forms of association. ${ }^{4}$ The core research problem is to understand how different conventions and organizational characteristics affect the tradeoffs among efficient allocation of resources, effective performance, and fairness. In particular, we are concerned with the implications of different ways of coordinating for reconciliation in the presence of increasing reach.

Articulation aimed at reconciling differences has an important and troublesome property. It cannot be cast as formalized mechanisms because the work itself involves justifying, designing, choosing, and enforcing just those very articulation procedures. This gives reconciliation work a kind of self-referential character. Sometimes, this appears in practice as debates over legitimacy, flagged by such phrases as "... it's for your own protection," and "... how dare you!" At other times, participants create workarounds that anticipate and adjust for inadequacies in local circumstances. For example, Gasser (1986) describes an engineering group that worked around defective software by running the program with false assumptions in a way that compensated for the design error. Sometimes, that is, it's necessary to go against policy, or standards, or even facts in order to get things done properly. This means that the brackets involved must be either flexible or subvertable. Above all, increasing reach forces us to consider reconciliation in situations where there is no common authority that can enforce a solution.

\footnotetext{
${ }^{4}$ For example, Williamson (1985, 1996), Ostrom (1990), Ostrom et al. (1994), Ellickson (1991), Rose (1994), and Sabel and Zeitlin (2004).
} 
I'll mention three ways of organizing reconciliation here: cross-cutting ties among participants, participant review, and patronage. These are not the only possible arrangements. These three organizational arrangements are neither market nor bureaucracy, and oppose the consequences of segregating and standardizing rationalization in many ways. They appear very frequently, and their possibilities and limits must be considered when thinking about brackets and information systems that embody them.

\subsubsection{Cross-Cutting Ties}

Reconciliation becomes more difficult when the problem lies not in the conduct of tasks directly, but in conflict among participants. Social scientists have often found that conflicts are constrained and softened by "crosscutting ties" (e.g., Gluckman 1965). That is, relations among friends, neighbors, relatives, and coworkers sometimes overlap considerably. When people in such situations come into conflict, there's a strong tendency for the quarrel to be softened by their mutual connections. In short, I'm less likely to do something rash to my neighbor's obnoxious pet if my neighbor is also my cousin and my business partner. Similarly, I can more easily trust someone far away with my property and my interests if we have kin and tradition in common. Landa (1994), for example, has provided an extensive study and comparative analysis of long-distance trading networks based on ethnic and kinship ties. More generally, each person participates in multiple networks simultaneously: kin, coworkers, friends, professional colleagues, neighbors, and so on. These networks often overlap in varying degrees. A problem in one network can sometimes be resolved by making use of connections through another overlapping network. ${ }^{5}$

In modern industry, organizational disputes are often mediated in this way. The process of side-stepping or working around official procedures often makes use of cross-cutting ties: Common aspects of identity, common history, common memberships in voluntary associations, friendship bonds, patron-client ties, and common professional commitments are all used to undercut or overcome formal "bureaucratic" restrictions. The work is done by taking someone out for coffee or a drink, by "letting down one's hair" in private conversation, by offering personal advice, perhaps indirectly. To put something "on the record" would be to destroy the possibil-

5 The analysis of interpersonal networks is a thriving part of sociology. Good introductions can be found in Watts (1999, 2003) and Burt (1992); Burt (2004) is particularly relevant to the problems discussed here. 
ity of using the cross-cutting tie to solve the problem. In such situations, if management is called upon to notice there's a problem, it's too late to solve it in that form-it's become another kind of problem. Sometimes then, cross-cutting ties conflict with official brackets of the kind described in manuals of policy and procedure. The effectiveness of using crosscutting ties to deal with a reconciliation problem comes from personal connections, cumulative experience with particular individuals, and common bonds. Systems that do not take this into account often appear rigid, hence ineffective. James Scott's analysis of economic modernization, for example, provides several striking examples of how things can go very wrong (Scott 1998).

On the other hand, cross-cutting ties are not an unmixed blessing; they can be, and often are, used to create or perpetuate differential access to benefits (Tilly 1998). For example, a group of friends may share knowledge of a valuable obscure resource (a good fishing site, for example) only among themselves. Or a group may capture the jobs in a particular industry or organization. Such ties are often used to obtain benefits outside the formal rules of bureaucratic or market organization and are thus reviled as corruption or cronyism by those excluded. But this is simply to say that there are no forms of social organization that are simultaneously efficient, effective, and fair by everyone's standards.

\subsubsection{Participant Review}

Participant review is another form of organization that has been receiving increasing attention. By participant review, I mean the aggregation of individual votes or opinions to reach a collective decision. Peer review of academic papers and proposals is a familiar example; political elections are another. Popularity contests, in which the declaration of a winner has no effect on collective action, are not matters of participant review in this sense. The basic procedure is to have people rate performances or candidates on some set of criteria. Ratings are then aggregated in some fashion to provide evaluative scores, which are then used to guide further action. Such rating systems are very flexible, because ratings can be weighted and aggregated in many ways. Moreover, collective policies can often be mapped onto the distribution of benefits in many ways as well. For example, certain categories of persons (e.g., senior citizens, children, criminals, veterans) may be given preferential access to some goods and services.

Participant review in some form is useful for evaluating performances, policies, and performers in situations where there is no centralized authority that can make decisions and enforce them. The process seeks to 
capture some of the benefits of market mechanisms without suffering their drawbacks. It differs from markets because it does not use money (or some other store of value) as a medium of exchange. Merit accumulated in one setting, therefore, cannot, in general, be transferred to other settings in any direct way. For example, the fact that I am widely recognized as the second-best football player in my household gains me nothing vis-à-vis my colleagues in the International Society for Intellectual History.

One sophisticated system of participant review is the moderation system used by the Slashdot Web site to evaluate postings and comments on them. ${ }^{6}$ Another such system is Bugzilla (http://www.bugzilla.org/about.html), the defect tracking system developed for the Mozilla open-source browser project. This system is described by Sandusky et al. (2004).

The usefulness of participant review as a device for aggregating preferences and distributing evaluations varies with circumstances. It is more likely to work in situations where the relevant evaluation criteria are clearly understood and reproducible. Similarly, where raters have a stake in the quality of the outcome, the work of rating will be taken more seriously.

As with any mechanism, participant review has its limitations and defects. Such mechanisms can often be gamed or subverted in various ways; doing this to Google page ranks has become a minor sport. Such systems can also lose or distort the significance of local commitments and constraints. One way of dealing with weaknesses in the participant review system is to make it recursive. That is, the system can be used to review the performance of the participant review task itself (and, by implication, the reviewers). Community pressure is thus brought to bear, in principle, on raters who are not careful and reliable. Another device is to limit the amount of rating that can be done and then reward raters who achieve good reputations with additional opportunities. Some of the most successful participant review systems, such as the one used by Slashdot, make use of these devices. Despite its drawbacks, participant review provides a flexible class of mechanisms for dealing with highly distributed

\footnotetext{
${ }^{6}$ Slashdot users of the site (http://slashdot.org; see also Benkler 2002) register, so they are known to the system. They then submit short articles of interest, and comment on those selected for posting. Posts and comments are evaluated by users. Those who provide good service to the system gain "karma" and additional moderation privileges. The system has many complexities (explained at http://slashdot.org/faq/com-mod.shtml\#cm600), including "metamoderation" (explained at http://slashdot.org/faq/metamod.shtml). As with all mechanisms of governance, it is often contested. It does appear to work.
} 
evaluation problems. Moreover, these mechanisms lend themselves reasonably well to support with information systems.

\subsubsection{Patronage}

A patronage system is comprised of personal relations between a few relatively powerful patrons and their relatively weak clients. ${ }^{7}$ Clients form a retinue or train that enhances the social position of the patron and provides a pool of resources for the participants to draw on. The patron provides access to wealth, glory, or other scarce goods, and perhaps some kind of political protection as well. Such a system of relations aggregates preferences, organizes reputations, and regulates resource flow. Patronage systems can thus serve as organizations for reconciling differences among participants. Alternatively, a patronage system may perpetuate differences that span rivalrous clienteles ("factions"). These disputes are often muted by cross-cutting ties, as in "Romeo and Juliet" or "West Side Story."

The relations in a patronage system are personal. They exist between a particular person as patron, and particular others as clients who may, in turn, have clients of their own. A patronage system is thus made up of chains of influence, obligation, and loyalty, rather than choices among fungible commodities. Their structure is thus a matter of unique histories, not a system of formal relations. The commitments that clients and patrons make to one another do not, in general, transfer to others.

We see patronage in operation nowadays wherever we find uniquely accomplished people who can command substantial resources in their own right. The most familiar examples are the star systems characteristic of many branches of the entertainment industries. Stars can build substantial entourages of supporters and assistants. They can also influence the hiring and investment decisions of firms and ventures in their industries. And, of course, they also benefit from the deference paid to celebrity.

The learned professions are a group of social worlds in which patronage relations flourish. Senior physicians, attorneys, professors, scientists, programmers, and engineers control considerable resources through their positions on review committees of all sorts and via the weight of their

\footnotetext{
7 Patronage has been analyzed in many contexts. Eisenstadt and Roniger (1984) provide a sociological overview. Schmidt et al. (1977) and Gellner and Waterbury (1977) are collections of studies from anthropology and political science. Clark (2000) argues convincingly that patronage was the dominant form of stratification in the 18th century. Biagioli (1993) provides a detailed analysis of the way patronage worked in early modern Italian science; Haskell (1980) discusses artistic patronage in the same period.
} 
recommendations, formal and informal. Eric Raymond has noted that patronage also plays a role in the support of highly distributed technical work such as open-source software development:

We may observe, in fact, that open-source firms hire star hackers for much the same reasons that universities hire star academics. In both cases, the practice is similar in mechanism and effect to the system of aristocratic patronage that funded most fine art until after the Industrial Revolution - a similarity of which some participants are fully aware. (Raymond 2001, p. 158)

The influence of an individual professional is acquired over time by converting personal reputation into referrals, appointments, assignment to plum projects, and other benefits. The personal reputation is acquired via participant review, formal and informal. Prestigious titles and awards serve as surrogate measures of reputation, but it is personal reputation that counts.

It isn't clear what effect the development of the Internet will have on patronage systems and their capacity to support and rationalize articulation work. One class of systems designed to support patronage has become quite popular: software used to organize social networks on the Web. There are now dozens of these services, and some of them apparently enroll many thousands of users. ${ }^{8}$ Another potential source of Web-based patronage is the system of links among blogs. Since popular blogs can increase traffic at other blogs by linking to them, a link from such a blog can be an important resource. Whether these nascent forms of patronage will come to be significant outside their immediate context is not yet clear.

\subsection{What Is to Be Done?}

Increasing reach means that many kinds of work are being changed in important ways. Traditional assumptions about the connections among tasks are changing, and new ways of working are being developed. As a result, the traditional assumptions that researchers have made about the relationships between technology and social organization now require careful rethinking. Most research in CSCW has been conducted on the assumption of relatively low reach. For the most part, case studies have looked at single organizations, or single units within organizations. CSCW research must consider how computing supports (or doesn't support, or might support) tasks in which the coworkers have never met, which have no particular

8 A partial list of current services is maintained at http://socialsoftware. weblogsinc.com/entry/9817137581524458/. 
location, and whose tools have substantial processing and communications capacities. This concluding section draws attention to some important research problems and suggests some strategies for development.

\subsection{The Changing Division of Labor in Computing Work}

One important aspect of increased reach is its implications for the organization of systems development work itself. For many years, it has been conventional wisdom that systems development requires two broad classes of expertise. On the one hand, it requires the skills of programming, system design, and other aspects of information systems work. On the other hand, it requires "domain knowledge," i.e., expertise in the substantive work to be served by the system under development. Traditionally, the notion of domain knowledge has included needed information about the organizations in which an application would be used, as well as the technical aspects of the work supported.

The development of the Internet has extended the kinds of information systems knowledge needed, but it has also led to the addition of a third kind of requisite expertise: local knowledge. Describing local circumstances in suitable ways is itself a difficult task. The describer must have access to the information and understand local circumstances well enough to represent them fairly and accurately without compromising needed confidentiality. At the same time, the describer must also be able to deal effectively with the systems development process. When the describers are system developers who have access to the ways local information is described, the problem is a manageable one. For systems of great reach, the system developers and the local describers are typically different people in different organizations. This can present insurmountable problems if relevant local circumstances cannot be described easily.

Designing and implementing brackets to support local articulation thus require a great deal of specialized local knowledge. In situations where the tasks may be based in different organizations with different cultures, located in different parts of the world, and subject to different regulatory regimes, specifying a satisfactory bracket will be a formidable task, one that will require different ways of organizing project development teams. 


\subsection{Strategies for CSCW}

It's clear that CSCW will be spending increased efforts on studying increased reach and its consequences. What help, at this stage, can CSCW research offer to systems developers concerned with concrete projects? I start with three assumptions. First, no information system will ever completely eliminate the needed local articulation work for any setting. Second, it will be possible to design and implement many kinds of system that will aid local articulation work in many kinds of setting. Third, brackets should fail gracefully. That is, a system should be able to "let go" and leave the coordination problems to local people when circumstances make it impossible for the mechanism to function adequately.

A number of pertinent research programs and computing innovations are currently underway-too many to permit a meaningful review here. These projects will provide a wealth of useful information to support the next round of research and experimental system development. Instead, I propose two broad rules of thumb: make customization easier and make reconciliation easier.

\subsubsection{Make Customization Easier}

The first suggestion is to make the work of customization easier. There are many ways to do this, and some of them are already part of standard practice. Most modern desktop applications, for example (accounting applications aside), let users adjust the visual properties of the user interface such as screen colors or type face and font size. Information systems should go far beyond this and support customizing local articulation tasks more generally.

Of course, no system can anticipate all the subtleties of a local context and its articulation problems. Rather than try to develop systems that will inevitably fail at this task, we should aim to turn local articulation experts into system developers. This can be done by building systems that support local bracket development much as integrated development environments support the development of programs. This idea rests on the distinction between domain expertise and local expertise. We can imagine local experts working with information systems experts to develop highly tailored systems using appropriate protocols.

One such system, for example, might support development of checklists to track completion of all the parts of complex projects. I am not thinking here of project management systems with their PERT charts, but rather a way to remind people and confirm that everyone has received needed 
resources, reminders, notices, and so on. As people in a particular situation discover that some participants need, say, more frequent reminders of approaching deadlines, this could easily be added as a heuristic. As experience accumulates in each situation, it becomes clearer to participants which things need to be checked more rigorously, and which can be safely ignored. The essential point is that the design of such a system must be modifiable by users, so that changing circumstances and idiosyncrasies can be reflected in the system easily and quickly.

More generally, studies should focus on ways in which the use of standardized procedures meets local articulation work. This will directly support development of information systems that reduce the total amount of articulation required, and simplify the remainder.

\subsubsection{Make Reconciliation Easier}

Understanding reconciliation and the limits to support it is certainly the most difficult problem facing CSCW research. There are several promising trends in the area. First, under the rubric of "social software," several different approaches to connecting people and facilitating negotiation are appearing and gaining considerable use. These are natural extensions of the collective messaging or "bulletin board" systems, which grew up in the 1980s. Major examples include group blogs, forums (in which participants post messages to public threads), and wikis (in which participants write and rewrite joint texts). Many of the features characteristic of each form are easily added to other forms, so we can expect a period of enthusiastic experimentation in which many ways of organizing, supporting, and managing discussion and negotiation are tried. The Bugzilla system mentioned above for reporting bugs in the Mozilla project (www.bugzilla.org/ about.html) has similarly evolved into a substantial mechanism for dealing with problems of reconciliation (e.g., Sandusky et al. 2004).

For obvious reasons, the development of new protocols (as opposed the use of established ones) provides a rich place to study reconciliation. An especially interesting example is the system of facilities supporting the Atom standard. Atom is a protocol designed to replace and extend the functionality of the RSS (and kindred) protocols for disseminating updates to Web sites. Development began in late 2003 and was carried out entirely via negotiation among interested parties on the Internet. In the course of this negotiation, a mix of supporting Web sites, blogs, and wikis was 
established to support the development work. ${ }^{9}$ As this sort of arrangement becomes more frequent, we will learn a great deal about supporting reconciliation effectively.

Systems to support varieties of participant review also hold a great deal of promise, both as practical applications and as vehicles for the study of research problems. What kinds of evaluation criteria work best, and with what kinds of rating system? Should the distribution of rating points be restricted and, if so, how? What sorts of access control are useful or counterproductive, and under what circumstances? When is meta-review effective, and when does it take more trouble than it's worth? These are the sorts of question that will guide additional research and development.

All these questions are predicated on the notion that $\mathrm{CSCW}$ is devoted to supporting (rather than controlling) the work that people do. There are situations in which computing support is not feasible or proper, and the best than can be done is to abandon the effort at support. Perhaps the most difficult technical challenge facing CSCW as a field is the development of effective means to recognize such situations and understand the limits to support.

\subsection{Conclusion}

Increasing reach based on the growth of the Internet has changed the way people work, the way we study work, and the role of computing in supporting work. This paper has focused on one important part of these changes: the nature of articulation work and the limits of computing support for it. Schmidt and Simone proposed the notion of coordination mechanism as a way to think about rationalizing articulation work in 1996. The impact of the Internet's growth since then requires that we think of articulation work (and hence, coordination mechanisms or brackets) in a more elaborate way.

It's important to distinguish between metawork (the work of organizing work) and local articulation work, the work of bringing together locally whatever is needed to carry out a task. Much metawork can be supported by computing technology in a fairly straightforward fashion. Moreover, the widespread adoption of many abstract standards and protocols makes it relatively easy to do so, at least by comparison with the pre-Internet situation.

${ }^{9}$ The specification is housed as a wiki at: www.intertwingly.net/wiki/pie/FrontPage; important blogs that track the project are at www.atomenabled.org/ and danja.typepad.com/fecho/. A mailing list for discussion can be found at www.imc.org/atom-syntax/index.html. 
Computing support in the form of brackets for local articulation presents a different and more complex set of problems. The central point about systems to support local articulation is that, wherever the flow of interaction goes beyond the limits of a protocol, effective computing support becomes very difficult to construct. This will typically be so, since local articulation is ordinarily concerned with negotiating limits and boundaries, rights and obligations, evaluation standards and entitlements, morals and emotional commitments. Attempting to organize such interaction with a formal system is simply an invitation to game or subvert the system. Systems to support local articulation should thus be devoted to supporting the negotiation and related efforts that make up such work rather than the work itself. Such systems are not likely to be brackets in the sense used here; they will guide the work only in the loosest sense.

\section{Acknowledgments}

I am grateful to Les Gasser, Arnold Goldfein, Christine Halverson, Carl Hewitt, Walter Scacchi, Kjeld Schmidt, and Carla Simone for helpful conversations over many years; to M. Sue Gerson, Christine Halverson, and an anonymous referee for helpful suggestions; to the editors for extraordinary patience; and to M. Sue Gerson for continuing support.

\section{References}

Becker HS (1982) Art Worlds. University of California Press, Berkeley, CA.

Benkler Y (2002) Coase's Penguin, or, Linux and the nature of the firm. Yale Law Journal 112: 1-72. http://www.benkler.org/CoasesPenguin.html.

Best M (1990) The New Competition: Institutions of Industrial Restructuring. Harvard University Press, Cambridge, MA.

Biagioli M (1993) Galileo, Courtier: The Practice of Science in the Culture of Absolutism. University of Chicago Press, Chicago.

Burt RS (1992) Structural Holes: The Social Structure of Competition. Harvard University Press, Cambridge, MA.

Burt RS (2004) Structural holes and good ideas. American Journal of Sociology 110: 349-399.

Campbell-Kelly M (2003) From Airline Reservations to Sonic the Hedgehog: A History of the Software Industry. MIT Press, Cambridge, MA.

Ceruzzi PE (2003) A History of Modern Computing, 2nd ed. MIT Press, Cambridge, MA.

Clark JCD (2000) English Society, 1660-1832: Religion, Ideology, and Politics During the Ancient Regime, 2nd ed. Cambridge University Press, New York. 
Coase RH (1937) The nature of the firm. Economica NS 4: 386-405. Reprinted in 1988 in The Firm, the Market, and the Law. University of Chicago Press, Chicago.

Cornes R, Sandler T (1996) The Theory of Externalities, Public Goods, and Club Goods, 2nd ed. Cambridge University Press, New York.

DiMaggio P (ed.) (2001) The Twenty-First-Century Firm: Changing Economic Organization in International Perspective. Princeton University Press, Princeton, NJ.

Eisenstadt SN, Roniger L (1984) Patrons, Clients, and Friends: Interpersonal Relations and the Structure of Trust in Society. Cambridge University Press, New York.

Ellickson RC (1991) Order Without Law: How Neighbors Settle Disputes. Harvard University Press, Cambridge, MA.

Garud R, Kumaraswamy A, Langlois RN (eds.) (2003) Managing in the Modular Age: Architectures, Networks, and Organizations. Blackwell, Malden, MA.

Gasser L (1986) The integration of computing and routine work. ACM Transactions on Office Information Systems 4: 205-225.

Gellner E, Waterbury J (eds.) (1977) Patrons and Clients in Mediterranean Societies. Duckworth, London.

Gerson EM, Star SL (1986) Analyzing due process in the workplace. ACM Transactions on Office Information Systems 4: 257-270.

Gluckman M (1965) Politics, Law, and Ritual in Tribal Society. Aldine, Chicago.

Haskell F (1980) Patrons and Painters: A Study in the Relations Between Italian Art and Society in the Age of the Baroque, 2nd ed. Yale University Press, New Haven, CT.

Hounshell DA (1984) From the American System to Mass Production, 18001932: The Development of Manufacturing Technology in the United States. Johns Hopkins University Press, Baltimore, MD.

Kanigel R (1997) The One Best Way: Frederick Winslow Taylor and the Enigma of Efficiency. Viking, New York.

Landa JT (1994) Trust, Ethnicity, and Identity: Beyond the New Institutional Economics of Ethnic Trading Networks, Contract Law, and Gift Exchange. University of Michigan Press, Ann Arbor, MI.

Landes DS (1983) Revolution in Time: Clocks and the Making of the Modern World. Harvard University Press, Cambridge, MA.

Lawrence PR, Lorsch JW (1967) Organization and Environment: Managing Differentiation and Integration. Harvard Business School Press, Boston, MA.

Malone T, Crowston K (1994) The interdisciplinary study of coordination. ACM Computing Surveys 26: 87-119.

NASA (1999) Mars climate orbiter mishap investigation board phase I report. National Aeronautics and Space Administration., Washington, DC. ftp:// ftp.hq.nasa.gov/pub/pao/reports/1999/MCO_report.pdf.

Ostrom E (1990) Governing the Commons: The Evolution of Institutions for Collective Action. Cambridge University Press, New York. 
Ostrom E, Gardner R, Walker J (1994) Rules, Games, and Common-Pool Resources. University of Michigan Press, Ann Arbor, MI.

Raymond ES (2001) The Cathedral and the Bazaar: Musings on Linux and Open Source by an Accidental Revolutionary. O'Reilly, Cambridge, MA.

Rose C (1994) Property and Persuasion: Essays on the History, Theory, and Rhetoric of Ownership. Westview Press, Boulder, CO.

Sabel CF, Zeitlin J (2004) Neither modularity nor relational contracting: Inter-firm collaboration in the new economy. Enterprise and Society 5: 388-403.

Sandusky RJ, Gasser L, Ripoche G (2004) Bug report networks: Varieties, strategies, and impacts in a F/OSS development community. To appear in Proceedings of the ICSE Workshop on Mining Software Repositories Edinburgh, Scotland, UK, May 25, 2004, pp. 80-84.

Schmidt K, Simone C (1996) Coordination mechanisms: Toward a conceptual foundation of CSCW system design. Computer Supported Cooperative Work 5: $155-200$.

Schmidt SW, Scott JC, Landé C, Guasti L (eds.) (1977) Friends, Followers, and Factions: A Reader in Political Clientelism. University of California Press, Berkeley, CA.

Scott JC (1998) Seeing like a State: How Certain Schemes to Improve the Human Condition Have Failed. Yale University Press, New Haven, CT.

Strauss AL (1987) The articulation of project work: An organizational process. Sociological Quarterly 29: 163-178.

Strauss AL (1991) Creating Sociological Awareness. Transaction Books, New Brunswick, NJ.

Strauss AL, Fagerhaugh S, Suczek B, Weiner C (1985) The Social Organization of Medical Work. University of Chicago Press, Chicago.

Sturgeon TJ (2002) Modular production networks: A new American model of industrial organization. Industrial and Corporate Change 11: 451-496.

Thompson JD (1967) Organizations in Action: Social Science Bases of Administrative Theory. McGraw-Hill, New York.

Tilly C (1998) Durable Inequality. University of California Press, Berkeley.

Trigg RH, Bødker S (1994) From implementation to design: Tailoring and the emergence of systematization in CSCW. Proceedings of the 1994 ACM Conference on Computer Supported Cooperative Work, pp. 45-54.

Watts DJ (1999) Small Worlds: The Dynamics of Networks Between Order and Randomness. Princeton University Press, Princeton, NJ.

Watts DJ (2003) Six Degrees: The Science of a Connected Age. Norton, New York.

Williamson OE (1975) Markets and Hierarchies. Free Press, New York.

Williamson OE (1985) The Economic Institutions of Capitalism: Firms, Markets, Relational Contracting. Free Press, New York.

Williamson OE (1996) Mechanisms of Governance. Oxford University Press, New York.

Wimsatt WC (1986) Developmental constraints, generative entrenchment, and the innate-acquired distinction. In Bechtel PW (ed.) Integrating Scientific Disciplines. Martinus Nijhoff, Dordrecht, pp. 185-208. 
Wimsatt WC (2001) Generative entrenchment and the developmental systems approach to evolutionary processes, In: Oyama S, Griffiths PE, Gray RD (eds.) Cycles of Contingency: Developmental Systems and Evolution. MIT Press, Cambridge, MA, pp. 219-238.

Yates J (1989) Control Through Communication: The Rise of System in American Management. Johns Hopkins University Press, Baltimore, MD. 